\title{
DESCARTES E O PROBLEMA DE PAPPUS
}

\author{
DECARTES AND PAPPUS PROBLEM
}

\author{
João Bosco Pitombeira de Carvalho ${ }^{1}$
}

\begin{abstract}
RESUMO
Este trabalho apresenta a solução do problema de Pappus por Descartes em seu La Géométrie, de 1637, embora ele já o tivesse resolvido em torno de 1632. Para isso, expomos, inicialmente, os preliminares de que Descartes necessitava para resolvê-lo, no Livro I de seu La Géométrie. Pappus tinha resolvido o problema no caso de 3 e 4 retas, e afirmou que nos casos com mais retas se obtinha, também, um lugar geométrico. Descartes não só resolve explicitamente o problema para 4 retas, mas indica quais são as soluções para os casos com mais retas. Os pré-requisitos para a solução do problema de Pappus consistem na construção de uma "álgebra de segmentos de retas", haja vista que Descartes afirma, logo no início de seu livro, que todo problema de geometria pode se reduzir ao estudo de alguns segmentos, estabelecendo relações entre eles. Essa álgebra de segmentos ensina como se somam, multiplicam e dividem segmentos, e como se extraem suas raízes quadradas. A solução de Descartes é uma aplicação brilhante de seu método para resolver problemas, exposto em seu Discours de la Méthode, do qual o La Géométrie é um apêndice. Com a resolução desse problema, Descartes pretende mostrar que seu método para atacar racionalmente problemas pode resolver, como ele afirma, qualquer problema de geometria. A resolução do problema de Pappus para qualquer número de retas mostrou claramente a força do método analítico de Descartes. Como Descartes se via como geômetra, ele está fazendo geometria em seu livro e não álgebra, ele constrói, posteriormente, o lugar geométrico.
\end{abstract}

Palavras-chave: René Descartes; Discours de la Méthode; La Géométrie; problema de Pappus; geometria analítica.

\begin{abstract}
We present Descartes's solution of Pappus's problem in the Géométrie of 1637, even though he had already solved it around 1632 . We present, first, the preliminaries Descartes needed to solve the problem. Pappus had solved the problem for the case of 3 or 4 lines and stated that in the general case one also obtained a locus. Descartes not only solved the problem of 4 lines, he sketches the solution for the cases with more lines. The preliminaries Descartes needs consist of a contruction on an "algebra of straight lines"; he states, right at the beginning of the Géométrie, that any geometry can be reduced of some segments by establishing relationships among them. This álgebra teaches how to add, subtract, multiply and divide segments of straight lines and how to find their square roots. Descartes solution is a brilliant application of his method to solve problems, exposed in his Discours de la Méthode, of which the La Géométrie is an
\end{abstract}

\footnotetext{
${ }^{1} \mathrm{PhD}$ em Matemática pela University of Chicago vinculado a Universidade do Estado do Rio de Janeiro UERJ, Rio de Janeiro, RJ, Brasil. Vínculo com Instituto de Matemática e Estatística - IME. Bolsista pesquisador da FAPERJ junto ao Programa PROFMAT do Instituto de Matemática e Estatística da UERJ. Endereço para correspondência: Rua Conde de Irajá, 386 - Ap. 501, Botafogo, Rio de Janeiro - RJ, Brasil, CEP: 22271-020. E-mail: jbpfcarvalho@gmail.com.
}

ORCID iD: https://orcid.org/0000-0002-0411-9565. 
appendix. Solving this problem, Descartes shows that his method to approach rationally a problem can solve, as he states, any problem in geometry. Descartes' solution of Pappus problem for any number of lines showed the power of his analytic method. Since Descartes viewed himself as a geometer, he is doing geometry and not álgebra in his book, he has to construct the locus whose equation he had found.

Keywords: René Descartes; Discours de la Méthode; La Géométrie; Pappus'problem; analytic geometry.

\title{
Introdução
}

Pappus de Alexandria (290(?) - 350(?)) publicou várias obras de matemática. Uma delas, o Coleção Matemática (Magno e Chaquiam, 2020), ficou conhecida pelos matemáticos europeus em 1566, devido a Commandino, que incluiu partes do livro em sua edição do Cônicas de Apolônio e que, também, em 1588 publicou uma edição das partes existentes da obra de Pappus. O livro foi muito estudado e discutido pelos matemáticos, pois nele Pappus resume, comenta e complementa boa parte da produção dos matemáticos gregos que o antecederam. Em 1631 Jacob Golius, holandês amigo de Descartes, chamou a atenção de Descartes para o problema.

Papppus tinha resolvido o problema no caso de 3 ou 4 retas. Descartes o resolveu completamente, descrevendo como as curvas obtidas variam de acordo com o número de retas do problema. Isso mostrou a força de seu método de atacar problemas, e lhe deu grande confiança.

\section{O problema de Pappus}

Em linguagem moderna, o problema de Pappus pode ser enunciado como segue: Dadas $n$ retas no plano, $L_{i}, n$ ângulos, $\theta_{i}$, n comprimentos, $m_{i}$, medidos sobre as retas que fazem os ângulos $\theta_{i} \operatorname{com}$ os $L_{i}$, e um segmento de reta $a$ (Veja a Figura 1 para ao caso de 4 retas). Pede-se o lugar geométrico dos pontos $C$ para os quais

\author{
No caso de 3 retas $m_{1}^{2}=m_{2} m_{3}$ \\ No caso de 4 retas $m_{1} m_{2}=m m_{4}$ \\ No caso de 5 retas $m_{1} m_{2} m_{3}=a m m_{5}$ \\ No caso de 6 retas $m_{1} m_{2} m_{3}=m_{4} m_{5} m_{6}$ \\ E assim sucessivamente.
}




\section{Figura 1}

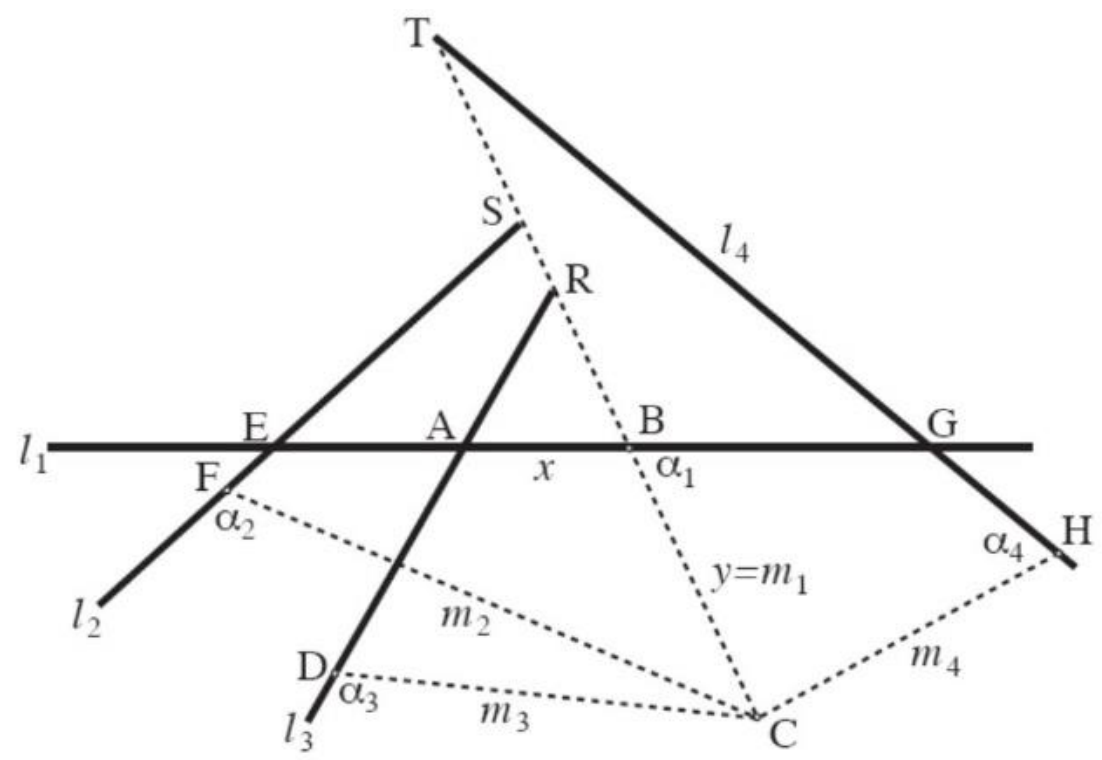

Quem desejar ver como Pappus enunciou o problema poderá consultar (Fauvel and Gray, 1990 pp. 340-342). Embora o problema fosse fácil de enunciar, Pappus somente conseguiu resolvê-lo em poucos casos, para 3 e 4 retas, apesar de ter afirmado que a solução do problema, no caso de cinco ou mais retas, seria sempre uma curva. Isso faz parte do encaminhamento da solução, pois poderia acontecer que o lugar geométrico fosse, por exemplo, um conjunto de pontos distribuídos pelo plano.

Para bem compreender a solução de Descartes e o papel que ela tem na obra matemática de Descartes, é necessário nos voltarmos para seu Discours de la Méthode (1637) e seu apêndice La Géométrie.

\section{O Discours de la Méthode de Descartes}

O grande objetivo do trabalho de Descartes era provar que a investigação racional podia gerar conhecimento, e seus trabalhos em matemática se destinavam, em parte, a mostrar como isso podia ser feito. Em verdade, para Descartes, a matemática era o paradigma da pesquisa racional, mas ele não menosprezou as ciências. Ele fez pesquisas em ótica, e esboçou uma teoria dos movimentos planetários que até os meados do século 18, pelo menos na França, era mais seguida que a teoria de Isaac Newton. 
Descartes sentiu a necessidade de levar a cabo um programa de remodelar todo o conhecimento. Ele se convenceu de que, para fazer isso, tinha que por em dúvida todas as ideias que adquirira desde sua infância. Vejamos o que diz Descartes:

Entre os ramos da filosofia estudei, quando mais jovem, a lógica, e entre os da matemática, a análise geométrica e a álgebra; três artes ou ciências que deveriam ter contribuído de alguma maneira para meus propósitos. Mas, examinando-as, chamou-me a atenção, no que diz respeito à lógica, que seus silogismos e a maior parte de seus outros métodos funcionam mais para explicar a alguém o que já sabemos, ou mesmo, [...] para falar livremente e sem discernimento do que não se sabe, e não para aprender coisas novas. Embora contenham muitos ensinamentos bons e verdadeiros, eles estão misturados com tantos que são ou danosos ou supérfluos que é quase tão difícil separá-los do que fazer surgir uma Diana ou uma Minerva de um bloco de mármore virgem. (Guinsburg, Romano e Cunha, 2010, pp. 74 - 75)

\section{Descartes continua}

Assim, no que diz respeito à análise dos gregos e à álgebra dos modernos, além do fato de que lidam com abstrações e especulações que também parecem não ter nenhuma utilidade, a primeira é sempre tão limitada à consideração de figuras que não pode propiciar a compreensão sem cansar muito a imaginação, e a última tão limitada a certas regras e a certos números que se tornou uma arte confusa e obscura que torna a mente perplexa, em vez de uma ciência que a educa. Por causa disso, julguei ser necessário procurar um outro método que, preservando as vantagens desses três, estivesse livre de seus defeitos. Finalmente, da mesma maneira que o grande número de leis frequentemente serve de desculpa para o vício, e um estado é mais bem governado com poucas leis que sejam estritamente obedecidas, eu julguei que em vez do grande número de preceitos que compõem a lógica, eu me contentaria com os quatro seguintes, desde que eu tomasse a resolução firme e inalterável de nunca violálos nem mesmo uma única vez. (Guinsburg, Romano e Cunha, 2010, pp. 75)

A partir de suas dúvidas, que regras Descartes estabeleceu como fundamentais para garantir pensar corretamente.

A primeira regra era nunca aceitar alguma coisa como verdadeira a menos que eu reconhecesse que ela certa e evidentemente o é: ou seja, evitar cuidadosamente toda precipitação e préjulgamento, e nada incluir em minhas conclusões a não ser que isso se apresentasse tão clara e distintamente à minha mente de maneira que eu não pudesse ter alguma ocasião para pô-las em dúvida. A segunda era dividir cada uma das dificuldades que eu tenha encontrado em quantas partes fosse possível e necessárias para melhor resolvêlas. A terceira era pensar de maneira ordenada quando empenhado na procura da verdade, começando com as coisas que fossem mais simples e mais fáceis de compreender, e gradualmente, aos poucos, avançar até os conhecimentos mais complexos, e supondo mesmo uma ordem entre os que não estavam necessariamente ordenados. A última era fazer em toda parte enumerações tão completas e revisões tão gerais de tal maneira que eu pudesse ter certeza de nada omitir. (Guinsburg, Romano e Cunha, 2010, pp. 78 -83)

\section{O Livro 1 do La Géométrie de Descartes}


Descartes principia o La Géométrie afirmando:

Qualquer problema em geometria pode ser facilmente reduzido a termos em que um conhecimento dos comprimentos de certos segmentos de reta é suficiente para sua construção. (Guinsburg, Romano e Cunha, 2010, pp. 491)

Para resolver um problema de geometria, ele propõe, essencialmente, o seguinte procedimento:

- 1 - Desenhe uma figura

- 2 - Identifique claramente o que você está tentando resolver

- 3 - atribua um nome a cada quantidade, conhecida ou desconhecida (por exemplo, $x, y, \ldots)$

- 4 - Escreva simbolicamente todas as relações entre essas quantidades

- 5 - Aplique várias técnicas a essas relações até que você tenha as incógnitas em equações que você consegue resolver.

Resumidamente, o Livro I consiste na representação dos números, na representação geométrica das soluções de uma equação do $2^{\circ}$ grau e da solução do problema de Pappus.

Inicialmente, Descartes assinala que trabalhar com segmentos de reta é tão fácil como trabalhar com números. Para isso, ele mostra como fazer a adição, diferença, multiplicação e divisão de segmentos e como extrair a raiz quadrada de um segmento.

1) A soma e diferença de segmentos não apresenta problemas. Em seguida, ele define o produto de segmentos da seguinte maneira. Na Figura 2, se $O C=1, O D=a, O F=b$, então, $O E=a b$.

\section{Figura 2}

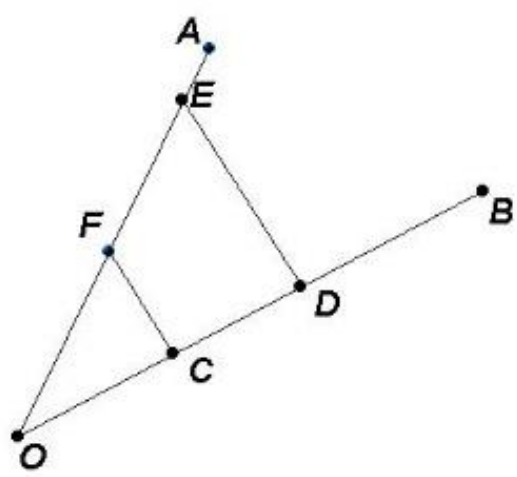


Na figura 3, se $O C=1, O D=a, O E=b$, então, $x=b / a$

\section{Figura 3}

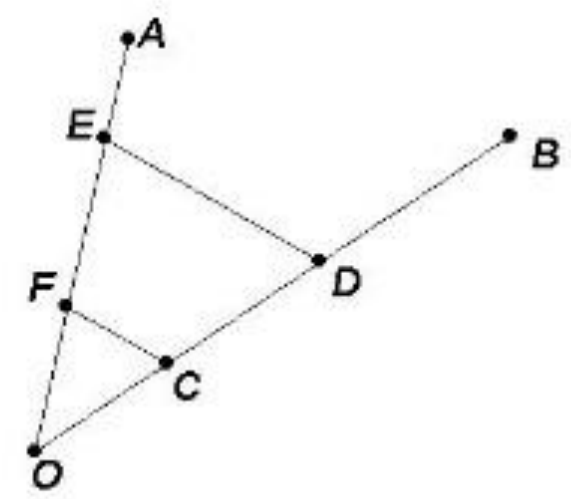

Observe que, para definir essas duas operações, Descartes tem que escolher uma unidade.

2) Em seguida, Descartes ataca o problema de como achar a raiz quadrada de um segmento.

\section{Figura 4}

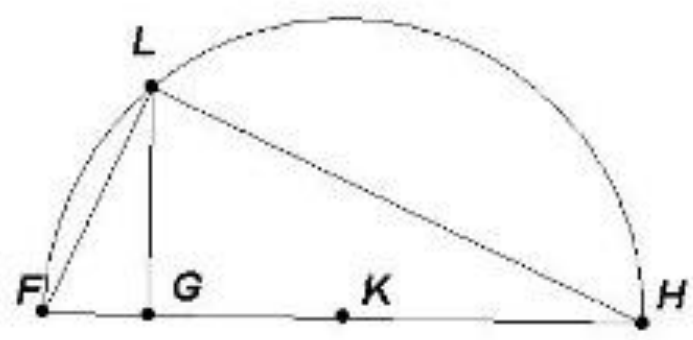

Dado o segmento $G H$, para achar sua raiz quadrada, $\sqrt{G H}$, Descartes procede como segue: Prolongue o segmento $G H$ até $\mathrm{F}$, de maneira que $F G=1$. Seja $K$ o ponto médio de $F H$. Trace o círculo de centro $K$ e raio $K H$ (Veja a Figura 4).

Seja GL a perpendicular ao diâmetro $F H$, por $G$. Então, pelas propriedades métricas em um triângulo retângulo, $G I=\sqrt{G H}$.

Fazendo isso, Descartes se liberta da exigência da homogeneidade da dimensão, preceito básico da geometria grega. 
3) Antes de atacar o problema de Pappus, Descartes mostra como resolver geometricamente equações do $2^{\circ}$ grau.

Descartes considera três tipos de equações:

$$
\begin{aligned}
& x^{2}=a x+b, \\
& x^{2}+a x=b, \\
& x^{2}+b=a x .
\end{aligned}
$$

a) Resolução da equação do tipo $x^{2}=a x+b$.

Para resolver esta equação (Figura 5), faça $O C=\frac{a}{2}, C D=\sqrt{b}, A D=x$.

\section{Figura 5}

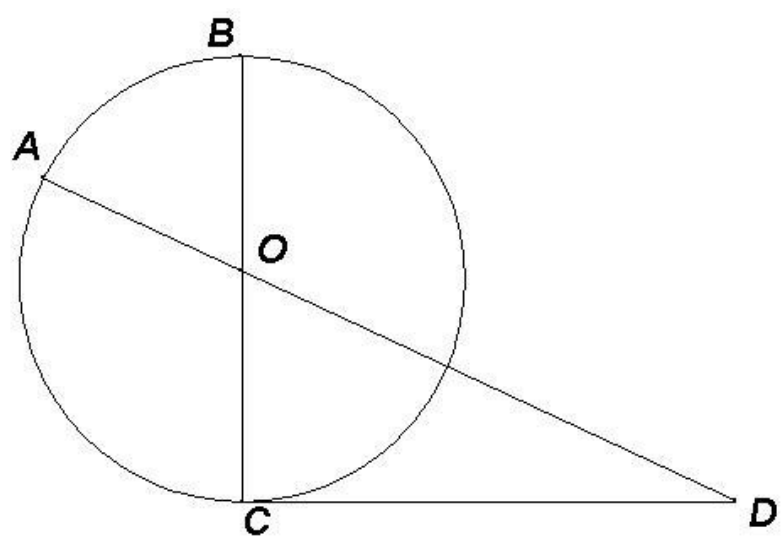

Temos que

$$
C D^{2}=D L \times A D
$$

ou seja,

$$
b=(x-a) x \rightarrow b=x^{2}-a x \rightarrow x^{2}=a x+b .
$$

b) Resolução da equação do tipo $x^{2}+a x=b$.

Na Figura 5, faça $L D=x$. Então

$$
x(x+a)=b \rightarrow x^{2}+a x=b .
$$

c) Resolução da equação do tipo $x^{2}+b=a x$.

Para resolver esta equação, Descartes procedeu da seguinte maneira (Figura 6). Seja $L M=\sqrt{b}$. Por $E$, levante a perpendicular $L N$, de comprimento igual a $\frac{a}{2}$. Por $M$ trace uma perpendicular a $L M$. Trace a circunferência de centro $N$ e raio $L N$ e sejam $Q$ e $R$ os pontos em que ela corta a perpendicular levantada a $L M$, por $M$. 


\section{Figura 6}

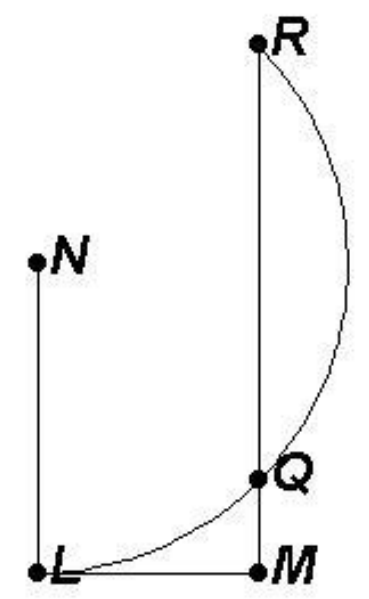

A fim de compreendermos melhor o raciocínio de Descartes, examinemos a Figura 7, construída complementando a Figura 6 com retas paralelas ou perpendicular a $L T$, como mostrado.

Figura 7

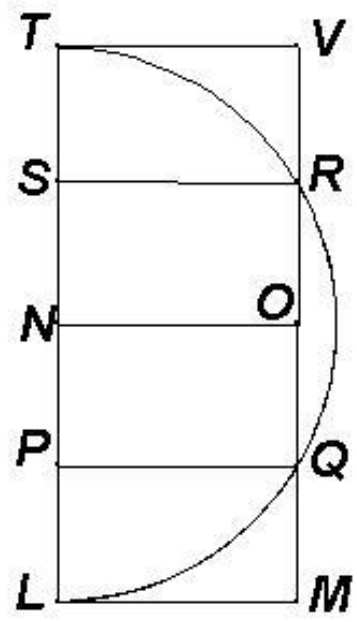

Faça $x=M R$. Por simetria, vemos que $M Q=R V$ e portanto $M Q=A-M R=$ $a-x$. Assim

$$
L M^{2}=M R \times M Q \rightarrow x(a-x)=b \rightarrow x^{2}+b=a x
$$

\section{A solução do problema de Pappus por Descartes}


Estamos agora prontos para entender a solução do problema por Descartes. Na

Figura 8 , façamos $A B=x$ e $m_{1}=y$.

\section{Figura 8}

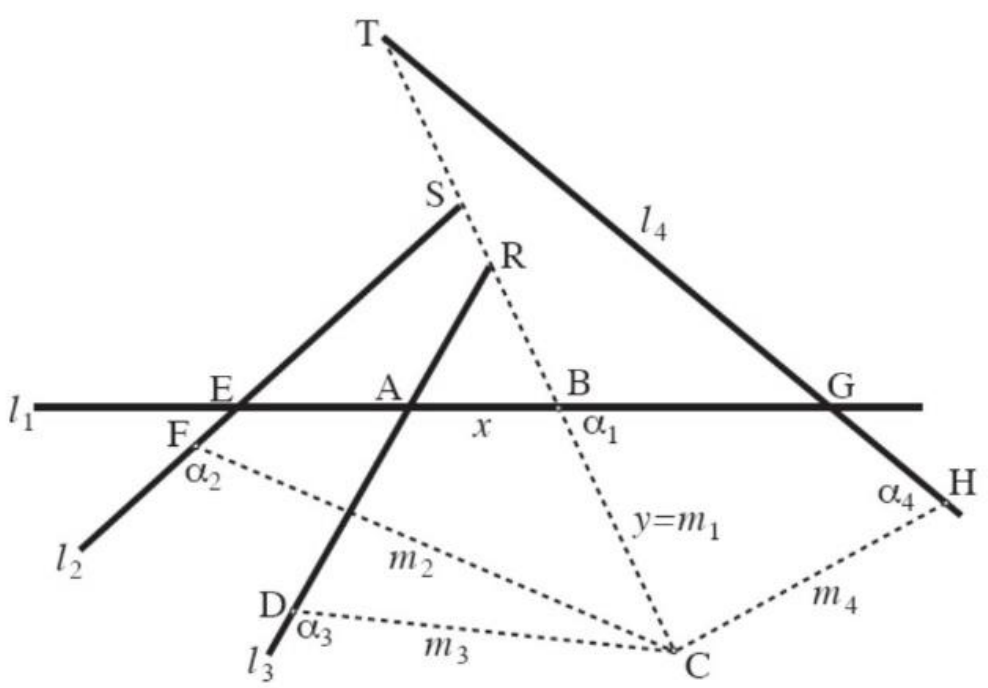

Para nós, acostumados com a "geometria analítica", criada por Descartes, certamente parece estranha a ausência dos familiares "eixos cartesianos". Em verdade, ao resolver um problema, Descartes procede da seguinte maneira, com suas palavras:

Assim, querendo resolver qualquer problema, deve-se primeiro considerá-lo como já feito, e dar nomes a todas as linhas que pareçam necessárias para construí-lo, como também para aquelas que são desconhecidas. Depois, sem considerar qualquer diferença entre essas linhas conhecidas e desconhecidas, deve-se percorrer o problema segundo a ordem que mostre, o mais naturalmente possível, de que modo elas dependem mutuamente uma das outras, até que se tenha encontrado um meio para exprimir uma mesma quantidade de duas maneiras, o que se chama uma equação. Pois os termos de uma dessas duas maneiras são iguais àquelas da outra. E deve-se encontrar tantas equações quantas as linhas supostas que eram desconhecidas. (GUINSBURG, ROMANO; CUNHA, 2010, p. 493)

No triângulo ARB faça $\frac{B R}{A B}=b$. Então,

$$
B R=b x
$$

E portanto $C R=y+b x$.

No triângulo DRC, faça $\frac{C D}{C R}=c$.

Assim,

$$
m_{3}=C D=c y+c b c .
$$


Agora, faça $A E=k, A G=l \mathrm{e}$

$$
\frac{B S}{B E}=d, \frac{C F}{C S}=e, \frac{B T}{B G}=f, \cdot \frac{C H}{T C}=g .
$$

Então, podemos escrever

$$
B E=x+k \rightarrow B S=d k+d x
$$

$\mathrm{e}$

$$
C S=y+d k+d x .
$$

E temos que $m_{2}=C F=e y+e d k+e d x$

Mas,

$$
\begin{gathered}
B L=l-x \rightarrow B T=f l-f x, \\
T C=y+f l-f x,
\end{gathered}
$$

e portanto

$$
m_{4}=C H=g y+g f l-g f x
$$

$\mathrm{e}$

$$
\begin{aligned}
& m_{1}=\mathrm{CB}=\mathrm{y} . \\
& m_{1}=y
\end{aligned}
$$

Devemos ter que

$$
m_{1} m_{2}=m_{3} m_{4}
$$

Ou seja,

$$
d e k y+d e x y+e y^{2}=b c f g l x+b c f g x^{2}+b c g x y+c f g l y-c f g x y+c g y^{2}
$$

Essa equação representa uma cônica, ou seja, é uma elipse, hipérbole ou parábola, dependendo dos valores dos coeficientes da equação.

Para nós, o problema está resolvido. Estamos tão habituados a identificar uma curva com sua equação que, pensando em uma delas, pensamos automaticamente na outra. Não para Descartes. Como geômetra que era, ele via a álgebra como uma ferramenta para resolver analiticamente problemas de geometria. O que realmente interessava era a geometria. Assim, Descartes tem que construir a solução das equações obtidas, o que já expusemos. Ele escolhe valores para y e chega então a equações do $2^{\circ}$ grau em $x$ que, em cada caso, ele resolve geometricamente.

\section{Considerações finais}


Com a solução do problema de Pappus, Descartes mostrou que seu método analítico era realmente poderoso. Aliás, no La Géométrie, ele afirma que o método permite resolver qualquer problema geométrico, já formulado ou ainda por formular. A solução do problema de Pappus, conseguida em poucos dias, em torno de 1632, Segundo Bos (1981, pp. 271-272) deu a Descartes grande confiança no poder de seu novo método analítico. Mostrou-lhe que o programa esboçado no Discours de la Méthode podia efetivamente gerar novos conhecimentos, livres de dúvidas e incerterzas.

\section{Agradecimentos.}

O autor agradece o auxílio financeiro da FAPERJ, com bolsa no Instituto de Matemática e Estatística da Universidade do Estado do Rio de Janeiro, sem o que este trabalho não poderia ter sido feito.

\section{Referências}

BOS, H. J. M.. On the representation of curves in Descartes' Géométrie. Archive for History of Exact Sciences, 24 (1981) pp. 295-338.

FAUVEL, John; GRAY, Jeremy. The History of Mathematics - a Reader. London: The MacMillan Press, 1990.

GUINSBURG, J; ROMANO, Roberto; CUNHA, Newton (orgs.). Descartes: Obras Escolhidas. São Paulo: Perspectiva, 2010.

MAGNO, Lucicleia Chagas; SOUZA, Ruan Wenderson de Oliveira; CHAQUIAM, Miguel. Pappus de Alexandria: contribuições à matemática. Boletim Cearense de Educação Matemática e História da Matemática. Fortaleza, 8(20), pp. 8-17, 2020

SMITH, David Eugene; LATHAM, Marcia L. (trads.). The Geometry of René Descartes. New York: Dover, 1954. 